\title{
Patterns of antifungal prescribing in general dental practice
}

\author{
R. J. Oliver, ${ }^{1}$ H. S. Dhaliwal, ${ }^{2}$ E. D. Theaker ${ }^{3}$ and M. N. Pemberton ${ }^{4}$
}

Objective To examine the current practice of antifungal prescribing by GDPs in the United Kingdom.

Design A postal questionnaire circulated to a random selection of 400 dentists.

Outcome measures The questionnaires were analysed and the responses expressed as absolute and relative frequencies.

Results Responses to the questionnaire were received from 297

$(74.3 \%)$ GDPs. Nystatin was the most popular choice of antifungal agent that GDPs would use, followed by miconazole, amphotericin B and

fluconazole. The likelihood of use of miconazole was positively linked to recent date of graduation. Lack of knowledge regarding

contraindications and problems with azole antibiotics was found in a significant minority of practitioners (36\%).

Conclusions The present study indicates that azole antifungal agents (especially miconazole) are becoming more widely used by GDPs, but that knowledge regarding potential problems with their use is suboptimal. Nystatin remains the most popular choice of antifungal agent.

Much attention has been focused on the prescribing of antibiotics recently. Such interest has largely examined antibacterial prescribing but there has been less work examining antifungal prescribing. Given that there is increasing evidence of Candida species becoming resistant to some antifungal agents, particularly the azole group, ${ }^{1}$ it is timely to examine the current prescribing habits in general dental practice.

Lewis et al. ${ }^{2}$ investigated the prescribing of antibiotics by general dental practitioners (GDPs) in the United Kingdom in 1987. Since that study, newer azole antifungals, namely fluconazole, have become available in general dental practice. However, the azoles, unlike the polyene antifungals, are absorbed from the gastrointestinal tract and thus have the potential for interactions with other drugs. They also have the potential to be hepatotoxic.

${ }^{1}$ Lecturer/Honorary SpR in Oral and Maxillofacial Surgery, ${ }^{2}$ GDP, formerly final year dental student, ${ }^{3}$ Lecturer in Oral Medicine, ${ }^{4}$ Consultant/Honorary Lecturer in Oral Medicine, Oral and Maxillofacial Sciences, University Dental Hospital of Manchester

Correspondence to: Dr Richard Oliver, University Dental Hospital of Manchester, Higher Cambridge Street, Manchester, M15 6FH

Email:richard.joliver@man.ac.uk

\section{Refereed Paper}

doi:10.1038/sj.bdj.4811354

Received 29.07.02; Accepted 1.08.03

$\odot$ British Dental Journal 2004; 196: 701-703
Over the last few years four antifungal drugs in a number of formulations, have been available for GDPs to prescribe under NHS regulations. ${ }^{3}$ Nysatin is available as pastilles, suspension and ointment for extra-oral use on the skin, while amphotericin is only available in lozenges and suspension for intra-oral use. Miconazole is available as an oral gel as well as an ointment and cream (combined with 1\% hydrocortisone) for use on the skin. Fluconazole capsules and suspension are available for oral/systemic use.

The aim of the present study was to establish the types and formulations of antifungal agents prescribed by a cross section of general dental practitioners during 1999/2000. We wanted to examine if prescribing habits were influenced by the time since graduation and the possession of postgraduate qualifications. Also, we wished to examine the awareness of practitioners regarding contraindications to, and problems with azole antifungals.

\section{MATERIALS AND METHODS}

The postal addresses of dental practitioners were obtained from the 1999 UK Dental Register. An initial total of 400 practitioners were selected by choosing every 74th entry in the register. Each of these dentists was sent an explanatory letter, a questionnaire (Fig. 1) and a reply-paid envelope. The questionnaires were encoded to enable follow-up of non-responders. To enable identification of GDPs, if the address was outside the UK or that of a hospital or community practice then the next name listed was chosen. In addition, all addressees were asked to return the uncompleted questionnaire if they did not work in general dental practice. If an uncompleted questionnaire was returned then a further questionnaire was sent to the next name listed on the register. Approximately 2 months later, a further questionnaire was sent to the non-responders.

A final letter and questionnaire was sent to non-responders with an additional 'tear-off' strip at the bottom of the letter asking the dentist to state why they did not want to participate.

Data from the received questionnaires were entered into a computer database and analysed and descriptive statistics were produced.

\section{RESULTS}

With the use of reminder letters and new questionnaires sent for those returned uncompleted, there were 297 responses, a response rate of $74.3 \%$. The majority of participants (36\%) graduated between 1980 and 1989, a quarter (27\%) graduated between 1970 


Fig. 1 The questionnaire
Please fill in the following questionnaire.
1. Do you have any postgraduate qualifications
$\begin{aligned} 1990-1999 \\ \text { 2. Year of qualification } \quad \text { YES } \square \text { NO } \\ 1970-1979 \\ 1960-1969 \\ \text { Before } 1960\end{aligned}$

3. Which antifungal agent(s) do you use to treat oral candidal infections? (Please tick the type of antifungal(s) and the form(s) you use)

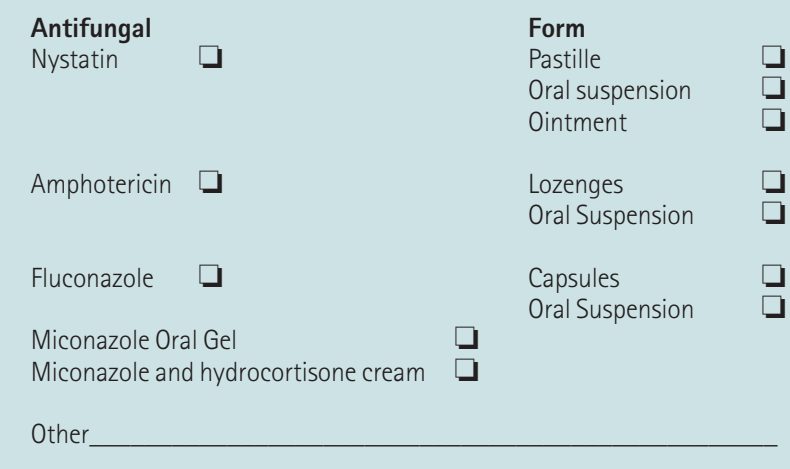

Nil $\square$

4. If you ticked miconazole are you aware of any contraindications to, or problems associated with, the use of this drug?

5. If you ticked fluconazole are you aware of any contraindications to, or problems associated with, the use of this drug?

Thank-you for your co-operation.

Please return the questionnaire in the stamped addressed envelope provided.

and 1979, with a similar proportion (25\%) graduating between 1990 and 1999. Only 9\% graduated between 1960 and 1969 with $3 \%$ graduating before 1959 . There were $19 \%$ of respondents who had postgraduate qualifications. Of the final requests for participation only four respondents returned the tear-off slip stating that they did not want to participate in the study.

The most popular antifungal prescribed (Table 1), in any form, was nystatin (81\%) followed by miconazole (45\%), amphotericin (37\%) and fluconazole (14\%) in that order. Many respondents (53\%) chose more than one type and/or form of antifungal; the nature of the questionnaire did not allow distinction between practitioners using simultaneous administration or practitioners using different antifungals for different manifestations of oral candidal infection in different patients.

\begin{tabular}{|c|c|c|}
\hline Antifungal agent & $n$ & $\%$ \\
\hline Nystatin & 240 & 81 \\
\hline Amphotericin & 110 & 37 \\
\hline Miconazole & 133 & 45 \\
\hline Fluconazole & 42 & 14 \\
\hline
\end{tabular}

With regard to the intra-oral preparations of nystatin available, pastilles were, by far, the most popular form with 85\% of those prescribing nystatin considering this formulation. Only 23\% of those prescribing nystatin would consider prescribing it in the form of an oral suspension. Lozenges were the most popular form of amphotericin prescribed (96\% of those prescribing amphotericin), with a much lower number considering prescribing the suspension form $(18 \%)$. Capsules were the most common form of fluconazole considered for prescription (82\% of those prescribing fluconazole) with oral suspension considered for prescribing by $27 \%$. Only 11 respondents considered prescribing fluconazole but not miconazole.

Surprisingly, only 90 respondents (31\%) indicated they prescribed any extra-oral antifungal preparations. Of these, most (78\%) prescribed nystatin ointment and 22\% miconazole with hydrocortisone.

Of those prescribing azole antifungals, 70\% (93/133) of those prescribing miconazole stated that they were aware of contraindications or problems associated with its use compared with $78 \%$ (33/42) of those prescribing fluconazole. However, of all 145 practitioners prescribing azole antifungals only 93 (64\%) showed awareness. Without exception, those who indicated an awareness of contraindications to fluconazole also indicated an awareness of contraindications to miconazole.

A striking result was the prescribing patterns of the azole antifungals, particularly miconazole, in relation to the year of qualification, particularly when adjusted according to the number of respondents in each group (Fig. 2). Clearly, recent graduates are more likely to prescribe miconazole. There was no relationship demonstrated between dentists prescribing choice and their possession of postgraduate qualifications.

Only 5\% of respondents cited other treatment options, which included chlorhexidine mouthwash, homeopathic remedies, Miltons solution for soaking dentures, yoghurt and treating the underlying cause.

\section{DISCUSSION}

The present study investigated the prescribing patterns for the currently available antifungal agents. The only previous study of this kind in the UK was undertaken in 1987 and reported in 1989 by Lewis et $a .^{2}$ and involved questioning about antibacterial and antiviral agents also. Only fluconazole was not available in the Dental Practitioners Formulary (DPF) at that time. ${ }^{4}$ However, in that study like the present study, nystatin was the most popular antifungal prescribed. There has also been an apparent increase in the proportion of practitioners prescribing miconazole in the present survey compared with 1987 and it has now become more popular than amphotericin B. Whilst the authors appreciate that in some instances the choice of antifungal agent is dependent on the form of candidosis being treated, the questionnaire was not prescriptive for the clinical form of candidosis, merely asking for all the antifungal agents routinely prescribed in general dental practice.

The polyene antifungals (nystatin, amphotericin B) act by binding to fungal cell membranes leading to leakage of the cellular components and cell death. They are poorly absorbed by the gastrointestinal tract and are widely used for the topical treatment of oral candidal infections. ${ }^{5}$ Resistance of Candida spp. to the polyene antifungals is rare. ${ }^{6}$ Nystatin has an unpleasant taste which may lead to poor patient compliance. ${ }^{7}$ To help overcome this, a sweetened pastille has been developed. Amphotericin was less frequently prescribed in the present survey than nystatin, but can be better tolerated than nystatin. ${ }^{7}$ Amphotericin (lozenges and cream) was reported as being equally as effective as fluconazole in one clinical trial of the treatment of denture stomatitis. ${ }^{8}$ Both polyenes can cause gastrointestinal disturbances.

Miconazole, an imidazole antifungal, inhibits fungal cytochrome P450 resulting in blocking cell membrane steroid synthesis. ${ }^{5}$ Miconazole was the first available azole and is also effective against gram-positive bacteria. It should be used with caution in those with liver disease and has significant drug interactions. Although used as a topical preparation for the treatment of oral candidosis, enough is absorbed into the circulation so it can still interact with drugs and has been reported to potentiate the effects of warfarin..$^{9}$ 


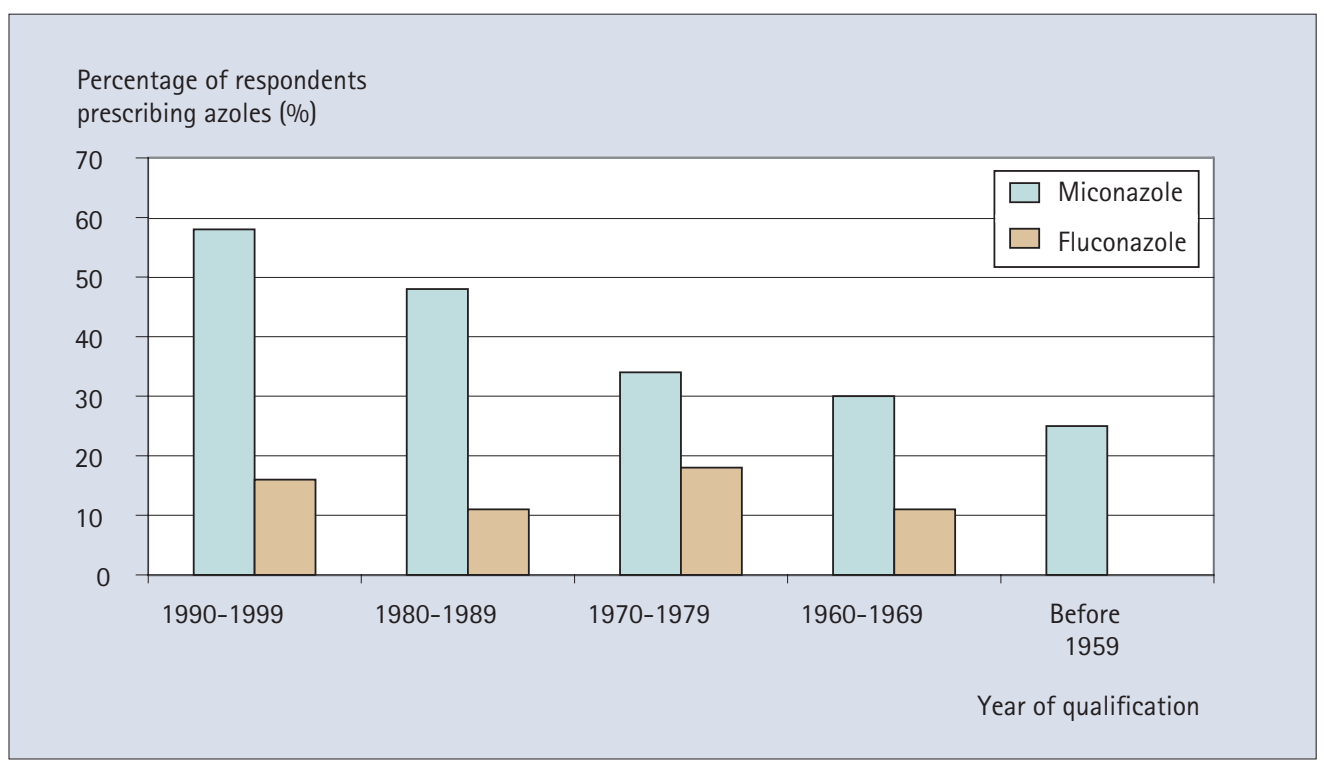

Fig. 2 Azole prescribing and year of qualification

Fluconazole is a more recent systemic antifungal agent, belonging to the triazole group, which has a long half-life and therefore can be administered in a single daily dose $(50 \mathrm{mg}$ ), which increases compliance. It has been widely used in the immunocompromised for the treatment of oro-pharyngeal candidosis when it is often used for prolonged periods; this has lead to the emergence of resistant strains of Candida albicans. ${ }^{10} \mathrm{~A}$ single high dose $(150 \mathrm{mg})$ formulation of fluconazole is now available as an over the counter medicine for the self-treatment of vaginal candidosis. Like miconazole, fluconazole has significant drug interactions and should be used cautiously in liver disease. Fortunately, the majority of respondents in the present survey stated they were aware of contraindications to the prescribing of azole antifungals. However, as there was no more detailed questioning beyond an awareness, it may be that the reasons why there are contraindications were not known.

Another triazole, itraconazole, is not available for dentists to prescribe but has a broader spectrum of activity than fluconazole. ${ }^{5}$ It has been reported to be as effective as fluconazole for the treatment of denture stomatitis in a pilot study. ${ }^{11}$

As well as the potentially serious side effects of fluconazole, another serious consideration is that of resistance of the Candida species to the drug. ${ }^{6}$ While reports of resistance so far have largely been in patients with HIV infection, there is a fear that the widespread use of fluconazole may alter the relative proportions of Candida species towards those which, unlike C. albicans, are intrinsically resistant. ${ }^{1} \mathrm{~A}$ recent American study has demonstrated no fluconazole-resistant strains of C. albicans in an otherwise healthy population with oral candidosis following treatment with fluconazole. ${ }^{12}$ Despite the availability of fluconazole as an overthe-counter medicine, there is not yet any evidence that this has been associated with an increase in resistance; however, there is no national surveillance scheme capable of detecting this. ${ }^{13}$

Other antimicrobial agents are available for topical administration in oral candidosis in the form of mouthwashes. Chlorhexidine, a bisguanide, is available in a wide variety of proprietary mouthwashes and is effective against fungal yeasts. ${ }^{14}$ It is recommended as a useful adjunct to the antifungal agents listed above ${ }^{15}$ although it should not be used simultaneously with nystatin as they interact and render each other ineffective. ${ }^{16}$ Another antimicrobial incorporated in some mouthwashes, cetylpyridinium chloride, has been reported, in vitro, to have greater fungicidal activity than chlorhexidine. ${ }^{17}$ The essential oil, tea tree oil, is available in proprietary products for the treatment of vaginal candidosis; an in vitro study ${ }^{18}$ has reported that it is effective against Candida albicans, however, a selection of essential oils were compared with each other but not with antimicrobial agents.

In summary, nystatin remains the most popular antifungal agent prescribed in the UK. There appears to be a trend towards the use of miconazole particularly among more recent graduates. While the majority of prescribers were aware of contraindications to the use of azoles, more than a third were not and this is a cause for concern.

The authors would like to thank the dentists who participated in this study.

1. Rex J H, Rinaldi M G, Pfaller M A. Resistance of Candida species to fluconazole. Antimicrob Agents Chemother 1995; 39: 1-8.

2. Lewis M A O, Meechan C, Macfarlane T W, Lamey P J, Kay E. Presentation and antimicrobial treatment of acute orofacial infections in general dental practice. BrDent J 1989; 166: 41-45.

3. British Medical Association and the Royal Pharmaceutical Society of Great Britain. Dental Practitioners' Formulary 2000-2002. London: 2000;

4. British Medical Association and the Royal Pharmaceutical Society of Great Britain. Dental Practitioners' Formulary 1986-1988. London: 1986;

5. Bagg J, MacFaralne T W, Poxton I R, Miller C H, Smith A J. Anonymous essentials of microbiology for dental students. Oxford: Oxford University Press, 1999; 105-116.

6. White T C, Marr K A, Bowden R A. Clinical, cellular and molecular factors that contribute to antifungal resistance. Clin Microbiol Rev 1998; 11: 382-402.

7. Budtz-Jörgensen E, Lombardi T. Antifungal therapy in the oral cavity. Periodontol 2000 1996: 10: 89-106.

8. Bissel V, Felix D H, Wray D. Comparative trial of fluconazole and amphotericin in the treatment of denture stomatitis. Oral Surg Oral Med Oral Pathol Oral Radiol Endod 1993; 76: 35-39.

9. Pemberton M N, Sloan P, Ariyaratnam S, Thakker N S, Thornhill M H. Derangement of warfarin anti-coagulation by miconazole oral gel. Br Dent J 1998; 184: 68-69.

10. Martin M V. The use of fluconazole and itraconazole in the treatment of Candida albicans infections: a review. J Antimicrob Chemother 1999; 44: 429-437.

11. Cross $L J$, Bagg J, Wray D, Aitchison T. A comparison of fluconazole and itraconazole in the management of denture stomatitis: a pilot study. J Dent 1998; 26: 657-664.

12. Haberland-Carrodeguas C, Allen C M, Beck F M, Buesching W J, Koletar S L, Sundstrom P. Prevalence of fluconazole-resistant strains of Candida albicans in otherwise healthy outpatients. J Oral Pathol Med 2002; 31: 99-105.

13. Hudson M M T. Antifungal resistance and over-the-counter availability in the UK: a current perspective. J Antimicrob Chemother 2001; 48: 345-350.

14. Meiller TF, Kelley J I, Jabra-Rizk M A, DePaola L G, Baqui A A M A, Falkler W A. In vitro studies of the efficacy of antimicrobials against fungi. Oral Surg Oral Med Oral Pathol Oral Radiol Endod 2001; 91: 663-670.

15. Ellepola A N B, Samaranayake L P. Adjunctive use of chlorhexidine in oval candidoses: a review. Oral Dis 2001: 7: 11-17.

16. Barkovv P, Attramadal A. Effect of nystatin and chlorhexidine gluconate on C. albicans. Oral Surg Oral Med Oral Pathol 1989; 67: 279-281.

17. Giuliana G, Pizzo G, Milici M E, Giangreco R. In vitro activities of antimicrobial agents against Candida species. Oral Surg Oral Med Oral Pathol Oral Radiol Endod 1999; 87: 44-49.

18. Hammer KA, Carson C F, Riley TV. In-vitro activity of essential oils, in particular, Melaleuca alternifolia (tea tree) oil and tea tree oil products, against Candida spp. J Antimicrob Chemother 1998; 42: 591-595. 